\title{
Rare species of bayrachny forests of the south of the European part of Russia
}

\author{
Tatyana Sokolova* \\ Federal research center southern scientific center RAS, 344006 Rostov-on-Don, Russia
}

\begin{abstract}
The article presents the results of long-term research of ravine oak forests of the Rostov region.The term 'bayrack' (ravine) forest is associated with the growing of forests of this type on the slopes of ravines in the forest-steppe zone. The conducted ecological and floral classification showed a high syntaxonomic diversity of ravine oak forests of the region. Forest communities are represented by 6 associations: Scorzonero ensifoliae-Quercetum Sokolova ex Semenishchenkov 2020; Vicio pisiformis-Quercetum roboris Semenishchenkov 2012; Pyro pyrastriQuercetum roboris Poluyanov 2012; Chamaecytiso ruthenici-Quercetum roboris Poluyanov 2012; Fritillario ruthenici-Quercetum roboris Onyschenko, Dyakova et Karpenko ex Goncharenko in Goncharenko et al. 2020, Melico pictae-Ulmetum minoris ass. nov. prov. from 2 classes: Carpino-Fagetea, Quercetea pubescentis and 2 unions: Aceri campestrisQuercion roboris and Scutellario altissimae-Quercion roboris. There are 67 rare plant species listed in the Red Books of the Rostov [1], Volgograd [2] and Voronezh [3] regions in the communities of these associations. Oak forests in the south of the European part of Russia significantly increase the biological diversity not only of the Don region, but also of the entire steppe zone of the country.
\end{abstract}

Oak forests in the south of the European part of the country are unique and rare ecosystems. All of them have been preserved only in specially protected natural areas, as well as in gully-beam systems (most of them are also protected). In the conditions of the steppe zone, it is the beams that are favorable forms of relief for the growth of the forest. The border location (on the border of natural zones, plains and hills) of the communities contributed to the development of high biological diversity. Taking into account the historical data on the distribution of forests in the south of the European part of the country: - the constant presence of thermophilic submediterranean: Buglossoides purpuro-caeruleum, Carex michelii, Dictamnus albus, Ligustrum vulgare, Melica picta; - south-siberian (Campanula glomerata, Carex pallescens, Fragaria viridis, Origanum vulgare, Rubus caesius, Veratrum lobelianum) species; - conclusions of Yu. D. Kleopov [4]: "The forests of the typological group under consideration, i.e. the forests of the "quercetalian order" Quercetalia, fall into two groups: western forests saturated with the subsurface type (before the Don River) and eastern forests-devoid of subsurface inhabitants, with a wide participation of South Siberian species"; it can be concluded that the forests of the southern

\footnotetext{
* Corresponding author: sta1562@yandex.ru
} 
European part of the country were a corridor between the forests of the Caucasus, Crimea and the forests of the European part of Russia.

The material for the work was geobotanical descriptions: 470 - author's, from the northern and central districts of Rostov (for 2007-2021), Bobrovsky, Bogucharovsky and Kantemirovsky-Voronezh (2021), Alekseevsky, Kumylzhensky and NekhaevskyVolgograd region; $(2011,2018,2021)$ and 777 - G. M. Zozulin (1960-1980s, the same regions). All geobotanical descriptions were entered into the TURBOWIN database [5]. The syntaxonomy is based on the ecological and floristic classification of the BrownBlanke direction [6-10]. According to the ecological conditions, the ravine oak forests of the studied territory are divided into mesophytic and thermophytic. The first ones occupy the northern slopes and the bottom of the large beam systems of the northern regions of all regions (Fig. 1). They correspond to the union Aceri campestris-Quercion Bulokhov et Solomeshch in Bulokhov et Semenishchenkov 2015, which unites Central Russian mesophytic broad-leaved forests without the participation of spruce. The remaining oak forests are thermophilic. For a long time, thermophilic forests of the European part of Russia, as well as Ukraine, were referred to the union Aceri tatarici-Quercion Zólyomy 1957, due to the weak study of thermophilic oak forests in the south of the European part of the country. Studies of oak forests of the foreststeppe zone (Voronezh, Kursk, Belgorod region, [7, 8] showed significant floral and ecological differences. Thus, the union Scutellario altissimae-Quercion roboris was identified, which unites the subxerophilic oak forests of the Pontic region of Ukraine and the south of Russia, found in the steppe zone and the southern part of the forest-steppe zone. Communities of thermophilic oak forests of the studied territory also correspond to this union (Table 1). They are common in the gullies up to the lower reaches of the Don River, and are also noted in the western and central regions of the Volgograd Region and in the above-mentioned regions of the Voronezh Region (Fig. 1).

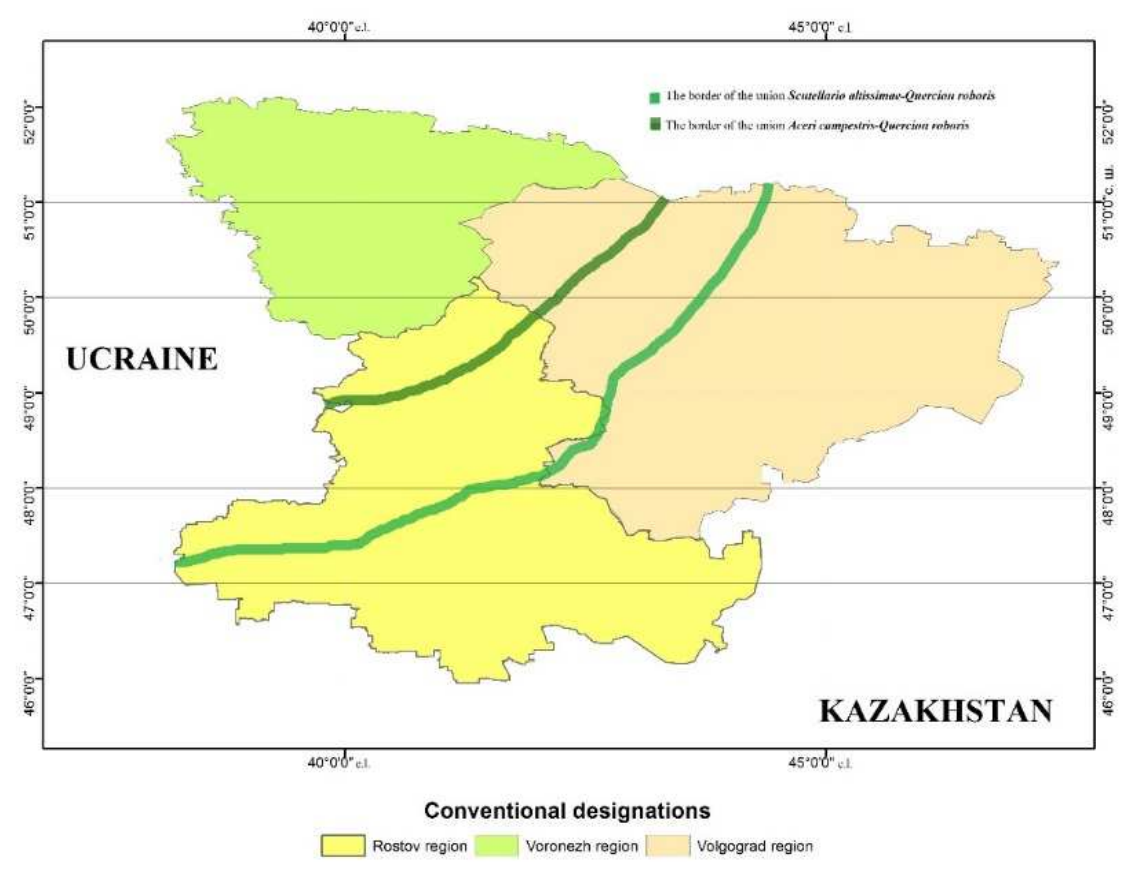

Fig. 1. Distribution of communities of unions Aceri campestris-Quercion roboris and Scutellario altissimae-Quercion roboris in the study area 
Table 1. Abbreviated differentiatial table of the associations of unions Aceri campestris-Quercion roboris and Scutellario altissimae-Quercion roboris

\begin{tabular}{|c|c|c|c|c|c|c|c|c|c|c|}
\hline \multicolumn{2}{|l|}{ Region } & \multicolumn{6}{|c|}{$\begin{array}{l}\text { The South of the European part } \\
\text { of Russia }\end{array}$} & \multicolumn{3}{|c|}{$\begin{array}{l}\text { Central } \\
\text { Russian } \\
\text { upland }\end{array}$} \\
\hline Number of descriptions & & 66 & 53 & 14 & 36 & 14 & 10 & 10 & 23 & 18 \\
\hline Syntaxon & & 1 & 2 & 3 & 4 & 5 & 6 & 7 & 8 & 9 \\
\hline Quercus robur & $\mathrm{A}$ & $\mathrm{V}$ & $\mathrm{V}$ & $\mathrm{V}$ & $\mathrm{V}$ & $\mathrm{V}$ & $\mathrm{V}$ & $\mathrm{V}$ & & $\mathrm{V}$ \\
\hline Quercus robur & $\mathrm{B}$ & II & II & & III & & & II & III & II \\
\hline \multicolumn{11}{|c|}{ Diagnostic species ass. Melico pictae-Ulmetum minoris } \\
\hline Ulmus minor & A2 & $\mathrm{V}$ & II & III & III & III & IV & & & \\
\hline Melica picta & $\mathrm{C}$ & IV & III & III & II & III & 2 & . & . & \\
\hline Aristolochia clematitis & $\mathrm{C}$ & III & II & II & II & I & . & . & . & . \\
\hline Ulmus minor & A & II & I & & I & & & 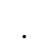 & 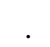 & \\
\hline
\end{tabular}

Diagnostic species ass. Vicio pisiformis-Quercetum roboris

Vicia pisiformis

Brachypodium sylvaticum

Phlomoides tuberosa

Carex rhizina

\begin{tabular}{|c|c|c|c|c|c|c|c}
$\mathrm{C}$ & I & V & I & I & I &. & V \\
C & II & III & I & II &. & IV & IV \\
C & I & III & II & II & II &. & IV \\
C &. & II &. &. &. &. & V
\end{tabular}

Diagnostic species ass. Chamaecytiso ruthenici-Quercetum roboris

Calamagrostis epigeios

Chamaecytisus ruthenicus

Malus sylvestris

Calamagrostis arundinacea

Moehringia trinervia

Peucedanum oreoselium

Hieracium umbellatum

Valeriana rossica

\begin{tabular}{|c|c|c|}
$\mathrm{C}$ & $\cdot$ & $\cdot$ \\
$\mathrm{B}$ & $\mathrm{I}$ & $\mathrm{I}$ \\
$\mathrm{A} 2$ & $\mathrm{I}$ & $\mathrm{I}$ \\
$\mathrm{C}$ & $\cdot$ & $\cdot$ \\
$\mathrm{C}$ & $\cdot$ & $\cdot$ \\
$\mathrm{C}$ & $\cdot$ & $\mathrm{I}$ \\
$\mathrm{C}$ & $\cdot$ & $\cdot$ \\
$\mathrm{C}$ & & $\cdot$
\end{tabular}

$$
\begin{array}{c|c|c|c|c|c|c|}
\text { IV } & \text { I } & \text { I } & \cdot & \cdot & \text { IV } & \text { II } \\
\text { V } & \text { I } & \text { I } & \cdot & \cdot & \text { V } & \text { I } \\
\text { III } & \text { I } & \cdot & \text { V } & \text { II } & \text { I } & \text { II } \\
\cdot & \cdot & \cdot & \cdot & \cdot & \text { IV } & \text { II } \\
\text { I } & \cdot & \cdot & \cdot & \cdot & \text { III } & \text { I }
\end{array}
$$

Pyrus pyraster

Pyrus pyraster

Astragalus glycyphyllos

Brachypodium pinnatum

Campanula rapunculoides

Diagnostic species ass. Pyro pyrastri-Quercetum roboris

Diagnostic species ass. Fritillario ruthenici-Quercetum roboris

Fritillaria ruthenica

Cerasus fruticosa

\begin{tabular}{|c|c|c|c|c|c|c|c|c|c}
$\mathrm{B}$ & II & II & II & V & III & V & IV & IV & IV \\
A & I & II &. & IV & I & $\cdot$ &. & IV &. \\
C & I & I & I & III & I & $\cdot$ & V &. & V \\
C &. &. &. &. &. &. &. & II & IV \\
C &. & I &. & II &. &. &. & I & V
\end{tabular}

\begin{tabular}{|l|l|l|l|l|l|} 
C & I & I & I & $\cdot$ & V \\
B & $\cdot$ & I & II & I & II
\end{tabular}

Diagnostic species ass. Scorzonero ensifoliae-Quercetum

Scorzonera ensifolia

Vincetoxicum hirundinaria

Viola suavis

Galeopsis tetrahit

Campanula rapunculus

Poa angustifolia

Daucus carota

\begin{tabular}{|c|c|c|}
$\mathrm{C}$ & $\cdot$ & $\cdot$ \\
$\mathrm{C}$ & $\mathrm{I}$ & $\mathrm{I}$ \\
$\mathrm{C}$ & $\mathrm{I}$ & $\mathrm{I}$ \\
$\mathrm{C}$ & $\mathrm{I}$ & $\mathrm{I}$ \\
$\mathrm{C}$ & $\mathrm{I}$ & $\mathrm{I}$ \\
$\mathrm{C}$ & $\mathrm{II}$ & $\mathrm{I}$ \\
$\mathrm{C}$ & $\mathrm{I}$ &.
\end{tabular}

\begin{tabular}{l|} 
II \\
I \\
. \\
. \\
II \\
I
\end{tabular}

$$
\begin{array}{c|c|c|c|c|c|}
\cdot & \cdot & \mathrm{V} & \cdot & \cdot & \cdot \\
\mathrm{I} & \cdot & \mathrm{V} & \mathrm{IV} & \mathrm{I} & \mathrm{III} \\
\mathrm{I} & \mathrm{II} & \mathrm{V} & \cdot & \cdot & \cdot \\
\cdot & \cdot & \mathrm{V} & \cdot & \cdot & \cdot \\
\mathrm{I} & \cdot & \mathrm{III} & \cdot & \cdot & \cdot \\
\cdot & \cdot & \mathrm{V} & \cdot & \mathrm{I} & \mathrm{II} \\
\cdot & \cdot & \mathrm{V} & \cdot & \cdot & \cdot
\end{array}
$$

Fraxinus excelsior

Acer campestre

Acer campestre

Fraxinus excelsior

Swida sanguinea

Euonymus europaea

\begin{tabular}{|c|c|c|c|c|c|c|c|c|c|} 
F & V & I & I & II & III &. & II & $\cdot$ &. \\
A2 & V & II & I & III &. & I & V &. & II \\
A & III &. &. & I & III &. &. & $\cdot$ &. \\
A2 & II & I & I & I & I & I & III & $\cdot$ &. \\
B & II & I &. & I & I &. & II & $\cdot$ & I \\
B & I & III & I & II & II &. & V &. & II
\end{tabular}


Acer campestre

Fraxinus excelsior

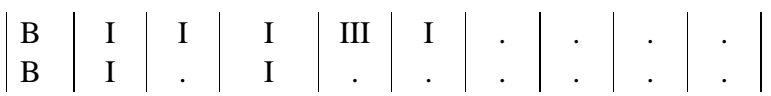

Diagnostic species union Scutellario altissimae-Quercion roboris

Crataegus rhipidophylla

Scutellaria altissima

Lamium maculatum

Acer tataricum

Dictamnus caucasicus

\begin{tabular}{|c|c|c|c|c|c|c|c|c|c} 
B & II & II & III & V & II & V & V & $\cdot$ & IV \\
C & II & I & I & II & I & $\cdot$ & $\cdot$ & $\cdot$ &. \\
C & I & I &. & II &. & $\cdot$ & $\cdot$ & $\cdot$ &. \\
B & I & V & IV & V & V & V & V & V & I \\
C &. & II & I & I & I &. &. &. &.
\end{tabular}

Diagnostic species order Carpinetalia betuli and class Carpino-Fagetea

Stellaria holostea

Geum urbanum

Dactylis glomerata

Poa nemoralis

Convallaria majalis

Tilia cordata

Viola mirabilis

Aegopodium podagraria

Campanula trachelium

Tilia cordata

Lathyrus vernus

Anthriscus sylvestris

Glechoma hederacea

Pulmonaria obscura

Acer platanoides

Stachys sylvatica

Carex muricata

Populus tremula

Heracleum sibiricum

Platanthera chlorantha

Corylus avellana

Scrophularia nodosa

Cystopteris fragilis

Asarum europaeum

Scilla siberica

Corydalis bulbosa

Polygonatum multiflorum

Corydalis marschalliana

Acer platanoides

Anemonoides ranunculoides

\begin{tabular}{|c|c|c|c|c|c|c|c|c|c|}
\hline $\mathrm{C}$ & $\mathrm{V}$ & I & III & II & II & & $\mathrm{V}$ & & II \\
\hline C & V & IV & II & V & III & V & IV & III & III \\
\hline C & III & V & II & IV & III & II & II & & III \\
\hline C & III & II & III & II & IV & I & V & III & IV \\
\hline C & III & II & II & II & I & V & III & III & II \\
\hline A & III & I & I & I & I & . & . & I & \\
\hline C & III & II & I & II & I & . & . & I & III \\
\hline C & III & I & . & I & . & V & . & I & III \\
\hline C & II & II & I & I & I & V & . & . & II \\
\hline A2 & II & I & I & . & I & . & IV & II & \\
\hline C & II & I & . & . & . & . & II & . & III \\
\hline C & II & I & . & II & II & . & II & II & III \\
\hline C & I & I & II & I & $\cdot$ & I & III & II & I \\
\hline C & I & I & I & I & . & II & III & . & III \\
\hline A & I & I & I & I & I & . & . & r & \\
\hline C & I & I & I & I & I & . & . & . & I \\
\hline C & I & II & I & I & I & . & . & . & $\therefore$ \\
\hline A2 & I & I & II & I & . & & . & II & II \\
\hline C & I & I & . & I & . & II & . & I & II \\
\hline C & I & I & I & . & . & . & . & . & \\
\hline B & I & . & I & . & . & . & . & I & II \\
\hline C & I & I & . & I & I & I & II & II & IV \\
\hline C & I & I & . & . & I & . & . & . & \\
\hline C & I & $\cdot$ & . & . & . & . & . & r. & II \\
\hline C & I &. & . & I & III & . & . & . & \\
\hline C & I & . & $\cdot$ & . & III & . & . & . & \\
\hline $\mathrm{C}$ & I & . & I & I & I & . & . & . & II \\
\hline C & I & I & I & . & II & . & . & . & \\
\hline A2 & I &. &. & . & . & . & V & II & I \\
\hline & I & . &. & . & II & . & 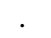 & . & \\
\hline
\end{tabular}

Diagnostic species order Quercetalia pubescenti-petraea and class Quercetea

Euonymus verrucosa

Polygonatum odoratum

Chelidonium majus

Viola hirta

Vincetoxicum scandens

Agrimonia eupatoria

Hypericum perforatum

Euphorbia semivillosa

Elymus caninus

Pyrethrum corymbosum

Prunus spinosa

\begin{tabular}{|c|c|c|c|c|c|c|c|c|c|} 
B & V & V & III & V & V & V & V & V & II \\
C & II & IV & III & III & III & III & II & V & I \\
C & II & I & . & III & III & V &. &. &. \\
C & II & II & II & II & II & III & IV & I & III \\
C & II & III & I & II & II & II &. &. &. \\
C & I & II & III & III & I & V & IV &. & IV \\
C & I & II & I & I & II & II & V &. & IV \\
C & I & I & II & II & II & III &. &. &. \\
C & I & I & I & I & I & I &. &. &. \\
C & I & III & II & I & II &. &. & I & IV \\
B & I & II & II & V & III &. & IV &. & II
\end{tabular}




Lactuca chaixii
Thalictrum minus
Clinopodium vulgare
Campanula bononiensis
Asparagus officinalis
Campanula persicifolia
Origanum vulgare
Lathyrus pisiformis
Stachys officinalis
Lapsana communis
Rhamnus cathartica
Vicia tenuifolia
Lactuca quercina
Pulmonaria mollis
Laser trilobium
Ligustrum vulgare
Asparagus tenuifolius
Genista tinctoria
Vicia cracca

\begin{tabular}{|c|c|c|} 
C & I & II \\
C & I & II \\
C & I & II \\
C & I & I \\
C & I & I \\
C & I & I \\
C & I & I \\
C & I & I \\
C & I & I \\
C & I & I \\
B & I & II \\
C & I & I \\
C & I & I \\
C & I & I \\
C & I & I \\
B & $\cdot$ & I \\
C & $\cdot$ & I \\
C & $\cdot$ & I \\
C & $\cdot$ & I
\end{tabular} \mid

\begin{tabular}{c|c|c|c|c|c|c|} 
II & III & IV & $\cdot$ & $\cdot$ & $\cdot$ & $\cdot$ \\
II & II & III & $\cdot$ & $\cdot$ & $\cdot$ & $\cdot$ \\
III & I & I & $\cdot$ & III & III & V \\
II & II & II & $\cdot$ & $\cdot$ & $\cdot$ & $\cdot$ \\
II & II & I & $\cdot$ & $\cdot$ & $\cdot$ & I \\
II & I & I & $\cdot$ & $\cdot$ & V & IV \\
II & I & I & $\cdot$ & $\cdot$ & I & II \\
I & I & I & $\cdot$ & $\cdot$ & $\cdot$ & $\cdot$ \\
I & I & I & $\cdot$ & III & IV & V \\
I & I & I & $\cdot$ & $\cdot$ & $\cdot$ & $\cdot$ \\
II & II & $\cdot$ & $\cdot$ & III & I & II \\
II & I & $\cdot$ & $\cdot$ & $\cdot$ & $\cdot$ & I \\
I & I & $\cdot$ & $\cdot$ & $\cdot$ & $\cdot$ & II \\
$\cdot$ & $\cdot$ & $\cdot$ & $\cdot$ & $\cdot$ & $\cdot$ & $\cdot$ \\
$\cdot$ & $\cdot$ & $\cdot$ & $\cdot$ & $\cdot$ & $\cdot$ & $\cdot$ \\
I & I & II & $\cdot$ & $\cdot$ & $\cdot$ & $\cdot$ \\
I & I & I & $\cdot$ & $\cdot$ & $\cdot$ & \\
II & $\cdot$ & $\cdot$ & $\cdot$ & $\cdot$ & $\cdot$ & II \\
$\cdot$ & I & $\cdot$ & I & $\cdot$ & $\cdot$ & I
\end{tabular}

Note: Symbols: The tiers of the stand: A - the first, A2-the second, B-shrub, C-grass. Constancy classes: I-1-20\%, II-21-40\%, III-41-60, IV-61-80, V-80-100\%.

Forest communities of bayrachny forests began their formation at the end of the Early Holocene [11], so there are many relict species here: Allium lineare L., Arum nordmannii Scott., Jasione montana L., Juniperus Sabina L., Ophioglossum vulgatum L., Ornithogalum boucheanum (Kunth) Asch. и др. Due to the borderline position of the forest distribution area, many species found themselves on the border of the range: Acer platanoides L., Adenophora lilifolia (L.) A. DC., Anthericum ramosum L., Archangelica officinalis (Moench) Hoffm., Asarum europaeum L., Bulbocodium versicolor (Ker-Gawler) Spreng., Campanula trachelium L., Cephalanthera damasonium (Mill.) Druce, Coccyganthe floscuculi (L.) Fourr., Dryopteris carthusiana (Vill.) H.P.Fuchs, Equisetum fluviatile L., Epipactis helleborine (L.) Crantz, Laser trilobium (L.) Borkh., Lathyrus niger (L.) Bernh., Lychnis chalcedonica L., Mercurialis perennis L.. The peculiarities of the formation, geographical location, and climatic conditions contributed to the formation of endemic species here: Aegonychon purpureo-caeruleum (L.) Holub, Delphinium sergii Wissjul. (D. schmalhausenii auct. non Albov.), Dictamnus gymnostylis Steven, Cotoneaster alaunicus Golitsin и др. In addition to the above, rare species are noted: Adonis volgensis DC. [Chrysocyanthus volgensis (DC.) Holub], Anemone sylvestris L., Anemonoides ranunculoides (L.) Holub, Asplenium ruta-muraria L., A. trichomanes L., Athyrium filixfemina (L.) Roth, Campanula altaica Ledeb., C. macrostachya Waldst. \& Kit. ex Willd., C. trachelium L., Clematis integrifolia L., Corydalis marschalliana (Pall. ex Willd.) Pers., $C$. solida (L.) Clairv., Delphinium puniceum Pall, Dryopteris filix-mas (L.) Schott, Echium russicum J. F. Gmel., Equisetum telmateia Ehrh., Eriosynaphe longifolia (Fisch. ex Spreng.) DC, Fritillaria ruthenica Wikstr., Galatella angustissima (Tausch) Novopokr., Hyacinthella leucophaea (C. Koch) Schur., Maianthemum bifolium (L.) F.W. Schmidt, Muscari neglectum Guss., Neottia nidus-avis (L.) Rich., Paeonia tenuifolia L., Paris quadrifolia L., Pedicularis dasystachys Schrenk, Platanthera bifolia (L.) Rich., P. chlorantha (Cust.) Reichenb, Prangos trifida (Mill.) Herrnst. \& Heyn, Primula veris L., Pteridium latiusculum (Desv.) Hieron. ex Fries, Polygonatum multiflorum Stev., Pulmonaria mollis Wulf. ex Hornem., P. obscura Dumort., Pulsatilla patens (L.) Mill., P. pratensis (L.) Mill. s. 1. м, Scilla siberica Haw., Symphytum tauricum Willd., Ulmus glabra 
Huds., Vinca herbacea Waldst. Et Kit., Vincetoxicum rossicum (Kleop.) Barbar. (Cynanchum rossicum Kleop.).

Acknowledgement. The publication was prepared within the framework of State Assignment for SSC RAS realization, Project No. AAAA-A19-119011190176-7.

\section{Reference}

1. Red Book of the Rostov region. Plants and fungi (Rostov, 2014)

2. Red Book of the Volgograd region. Plants and other organisms (Voronezh, 2017)

3. The Red Book of the Voronezh Region. Plants. Lichens. Mushrooms. (Voronezh, 2018)

4. J. D. Kleopov, Analysis of the flora of deciduous forests of the European part of the USSR. (Naukova dumka, Kiev, 1990)

5. S. M. Hennekens, TURBO(VEG). Software package for imput, processing, and presentation of phytosociological data (Lancaster, IBN-DLO, 1996)

6. J. Braun-Blanquet, Pflanzensoziologie. Grundzüge der Vegetationskunde (SpringerVerlag, Wien, 1964)

7. L. Mucina, H. Bültmann, K. Dierßen, J-P. Theurillat, T. Raus, A. Čarni, K. Šumberová, W. Willner, J. Dengler, RG. García, M. Chytrý, M. Hájek, R. Di Pietro, D. Iakushenko, J. Pallas, FJA. Daniëls, E. Bergmeier, A. Santos Guerra, N. Ermakov, M. Valachovič, JHJ. Schaminée, T. Lysenko, YP. Didukh, S. Pignatti, JS. Rodwell, J. Capelo, HE. Weber, A. Solomeshch, P. Dimopoulos, C. Aguiar, SM. Hennekens, L. Tichý. Applied Vegetation Science 19(1) (2016)

8. I. Goncharenko, Y. Semenishchenkov, J. Tsakalos., L. Mucina. Biology, 75 (1) (2020)

9. Yu. A. Semenishchenkov, A.V. Poluyanov, Rast. Ross. 24 (2014)

10. T. A. Sokolova, Actual problems of the humanities and natural sciences 3(26) (2011)

11. G. M. Zozulin, Forests of the Lower Don (Rostov-on-Don, 1992) 